Sección uno: Ensayo

Radiografía de la innovación educativa en el Siglo XXI

\title{
Una experiencia de innovación docente: el debate académico en Administración de Empresas ${ }^{1}$
}

A teaching innovation experience: the academic debate in business management

\author{
Francisca Orihuela-Gallardo \\ Universidad de Cádiz \\ francisca.orihuela@uca.es \\ Cristina Sierra-Casanova \\ Universidad de Cádiz \\ cristina.sierra@uca.es
}

\section{Resumen}

Este trabajo muestra una experiencia en la que se ha planteado el debate académico como elemento motivador y dinamizador en el aula, además de canalizador en la adquisición y mejora de las competencias asociadas a la asignatura “Administración de Empresas” del Grado en Relaciones Laborales y Recursos Humanos de la UCA, en un contexto caracterizado por un alumnado que muestra apatía, falta de asistencia y participación en clase y con algunas dificultades a la hora de abordar y afrontar la realización de trabajos genuinos y realizados en equipo. Como resultados cabe destacar el interés despertado entre el alumnado, mostrando una alta satisfacción y una mejora tanto en su percepción sobre el nivel de competencias adquiridos como en las calificaciones finales obtenidas.

Palabras clave: Innovación docente, debate universitario, competencias transversales, rendimiento, asistencia a clase, satisfacción

${ }^{1}$ Recibido: 20/01/2020 Evaluado: 09/02/2020 Aceptado: 19/02/2020 


\begin{abstract}
This paper explains the experience of using academic debate as a motivating and invigorating element in class. Academic debate has also been used as a catalyst for the competences acquisition and improvement in connection to the "Business Management" course in the Labour Law and Human Resources degree in the "Universidad de Cadiz". The context of said course is apathy, low class attendance and lack of participation. The students have historically shown difficulties when addressing and doing genuine and team works. As main results are worth to mention the increase in the students' interest, who indicate a high satisfaction and an improvement in both their own perception about their new acquired competences and the final grades.
\end{abstract}

Keywords: Teaching innovation, academic debate, transversal competences, class attendance, motivation, satisfaction

\title{
Introducción
}

La puesta en marcha de los nuevos grados universitarios dentro del marco del Espacio Europeo de Educación Superior ha traído consigo cambios en el proceso de enseñanzaaprendizaje con importantes repercusiones en el aula. El enfoque hacia la adquisición y desarrollo de competencias, que pone el foco en el proceso de aprender a aprender y no en la mera transmisión de conocimientos, le ha asignado un papel más activo al alumnado y ha transformado el rol del profesorado en el aula (Marqués, 2001; Rianudo et al, 2006). Así, los docentes universitarios vienen implantando metodologías innovadoras que incluyen la realización de actividades en las que la presencialidad del alumno en clase es un elemento esencial, variable que adquiere una gran relevancia. Así, Rodríguez y Herrera (2009) señalan la importancia de la asistencia a las clases presenciales, ya sean teóricas o prácticas, para la superación de las asignaturas y Bernardo et al (2015) apuntan la influencia de la asistencia a clase en las tasas de abandono de los estudios universitarios y en el rendimiento académico en la universidad, presentando mejor rendimiento y mayores probabilidades de permanencia los que asisten con más frecuencia a clase.

Estos cambios metodológicos vienen acompañados, en gran medida, por la utilización de nuevos recursos tecnológicos. El uso de las tecnologías de la información y comunicación (TICs) en la docencia ha proporcionado una fuente permanente, rápida y fiable para la búsqueda y tratamiento de información y datos, ha abierto nuevos canales de comunicación entre pares y con los docentes y ha abierto nuevas plataformas para el desarrollo de trabajos colaborativos (Marqués, 2001).

Sin embargo, como señala Marqués (2001), la mayor disponibilidad de las TICs no implica necesariamente un cambio sustancial en las metodologías empleadas por los docentes ya que "antes el profesor daba sus clases magistrales con el apoyo de la pizarra y los alumnos presentaban sus trabajos y exámenes escritos a mano o a máquina; ahora el profesor da sus clases magistrales con Power Point, los estudiantes presentan sus trabajos en Word y a veces los exámenes son pruebas objetivas ante un ordenador” (p.91). Es precisamente este hecho, 
unido al desarrollo de los denominados campus virtuales y la lectura que hacen de ello el alumnado, una de las posibles causas de la falta de asistencia a clase que venimos observando en los últimos años.

Desde nuestra experiencia como docentes en la asignatura Administración de Empresas del Grado en Relaciones Laborales y Recursos Humanos (GRRLLyRRHH) en la que se circunscribe este trabajo, hemos detectado dos aspectos que, sin duda, repercuten en el desempeño del alumnado en dicha asignatura. En primer lugar, una importante apatía y desmotivación por aprender, y el esfuerzo necesario que ello implica, así como una importante desorientación hacia cuáles son sus metas personales y profesionales. En este sentido hay que señalar que esta es una titulación en la que el alumnado es moderadamente vocacional, el Autoinforme de Seguimiento del Título (Facultad de Ciencias del Trabajo, 2019) indica que alrededor del $40 \%$ del alumnado de nuevo ingreso no la ha elegido como primera opción por lo que muchos de ellos no son capaces de identificar las salidas profesionales de la misma. Todo ello se ha traducido en una progresiva falta de asistencia, sobre todo a las clases teóricas y aquellas otras actividades que no tengan una traducción directa de notas en la evaluación final. En segundo lugar, a pesar del gran uso y alto conocimiento que muestran sobre el manejo de las TICs, presentan deficiencias importantes en la gestión, selección y tratamiento de la información. Como consecuencia de ello, presentan una importante barrera para diseñar trabajos genuinos y originales con soluciones a necesidades reales actuales de su entorno inmediato, a trabajarlos en equipo y a transmitir y comunicar en público los resultados de dicho trabajo, aspectos asociados a competencias generales del título y competencias transversales que la Memoria Verificada del Título (Facultad de Ciencias del Trabajo, 2019) asigna a esta asignatura. Las competencias estudiadas en este trabajo se recogen en la tabla 1.

Tabla 1: Competencias específicas, generales y transversales analizadas

\begin{tabular}{|l|c|l|l|}
\hline Id. Grado & Nomenclatura & \multicolumn{1}{|c|}{ Descripción Competencia } & \multicolumn{1}{|c|}{ Tipo } \\
\hline \multirow{2}{*}{ CG1/CT3 } & C1 & $\begin{array}{l}\text { Capacidad de comunicación oral usando } \\
\text { terminología y técnica adecuada }\end{array}$ & $\begin{array}{l}\text { General/Transver } \\
\text { sal }\end{array}$ \\
\cline { 2 - 4 } & C2 & $\begin{array}{l}\text { Capacidad de comunicación escrita usando } \\
\text { terminología y técnica adecuada }\end{array}$ & $\begin{array}{l}\text { General/Transver } \\
\text { sal }\end{array}$ \\
\hline CG3 & C3 & $\begin{array}{l}\text { Capacidad para seleccionar y gestionar } \\
\text { información y documentación laboral }\end{array}$ & General \\
\hline CT7 & C4 & Capacidad para la toma de decisiones & Transversal \\
\hline CT8 & C5 & Destreza para el trabajo en equipos & Transversal \\
\hline
\end{tabular}




\begin{tabular}{|l|c|c|c|}
\hline Id. Grado & Nomenclatura & \multicolumn{1}{|c|}{ Descripción Competencia } & Tipo \\
\hline CE5 & C6 & $\begin{array}{l}\text { Capacidad para conocer e identificar los } \\
\text { conocimientos teóricos propios de la } \\
\text { Organización y Dirección de Empresas }\end{array}$ & Específica \\
\hline
\end{tabular}

Fuente: Memoria Verificada del GRRLLyRRHH (Facultad de Ciencias del Trabajo, 2019)

Esta realidad, junto a la necesidad de emplear recursos didácticos "que favorezcan el aprendizaje autónomo, reflexivo, crítico y cooperativo” (Rodríguez y Herrera, 2009:9), nos lleva a hacer un replanteamiento de la metodología aplicada que posibilite la obtención de cuatro objetivos. El primer objetivo pretende fomentar la comprensión de forma consciente por parte del alumnado de las competencias específicas, generales y transversales de la asignatura, así como su adquisición y desarrollo. El segundo la mejora de la capacidad del alumnado en el proceso de identificación, búsqueda de información y comunicación de ideas interesantes relacionadas con la empresa y su entorno. El tercero incrementar las tasas de rendimiento de la asignatura, así como las tasas de asistencia y participación en clase. Por último, el cuarto objetivo, implicar al alumnado en el proceso de valoración y evaluación de esta metodología a través de su satisfacción y las propuestas de mejora. En este contexto, se plantea el debate académico como un recurso docente innovador con capacidad para fomentar la asistencia regular a clase y la participación activa en la misma, el desarrollo de las competencias generales, transversales y específicas y la mejora en el rendimiento académico del alumnado participante.

\section{El debate académico como recurso de innovación docente}

El debate académico, o debate universitario, es un recurso didáctico que viene siendo muy utilizado en las universidades de todo el mundo y que permite desarrollar las competencias transversales y generales del alumnado (Ahumada et al, 2015; Sánchez, 2007). El debate requiere un análisis crítico del tema tratado y exige al estudiante, no solo que opine y defienda sus argumentos, sino que los defienda ante otros, lo que mejorará su capacidad de comunicación y de entender las posiciones contrarias. De esta manera, el debate favorece el pensamiento crítico, elemento fundamental en el proceso de construcción de conocimiento propio (Leitáo et al, 2016). Sánchez (2007:2) lo define como “cualquier proceso de intercambio dialéctico entre dos o más partes con el objetivo final del voto favorable o aprobación de un tercero”. Este es el enfoque desde el que planteamos los debates en el aula y, por ello, se ha realizado una adaptación de la metodología propia de los debates académicos a fin de conseguir la implicación y participación de todo el alumnado asistente al debate. Para ello, se han organizado como se expone a continuación.

El alumnado forma equipos de trabajo con cuatro componentes. Cada equipo trabajará sobre un Proyecto o Idea, proponiendo un tema, problema o noticia que consideren inspiradora y de interés tanto para ellos como para su audiencia (compañeros de clase), que sea actual, reflejo de la realidad y relacionado con la asignatura de Administración de Empresa, es decir, cualquier tema relacionado con la empresa y su entorno, entendiendo la empresa cómo sistema abierto. La responsabilidad de la elección del tema, por tanto, recae sobre los 
componentes del equipo, ya que este debe ser un tema que les guste y les motive a ellos y a sus compañeros de clase. Como señalan Rianudo et al (2006), para obtener un buen resultado no es suficiente que el alumnado sea capaz de lograrlo, sino que su interés, dedicación y esfuerzo son elementos fundamentales.

Los equipos formados son totalmente autónomos para organizar y distribuir las tareas necesarias para poder llevar a cabo el debate en la clase. Lo que sí está definido con antelación es la estructura de la sesión de debate en sí. En concreto, la distribución de los roles de los cuatro componentes del equipo es la siguiente:

- Alumno 1 (Introductor): Realiza la introducción o exposición inicial del tema elegido tras lo cual lanzará la pregunta de debate a la clase que será la que centre la discusión. La audiencia se posicionará a favor o en contra de la pregunta a tratar en el debate antes de conocer la argumentación y las refutaciones a favor o en contra. El alumno 1 anotará en la pizarra el número de personas a favor y en contra inicialmente (2 min.).

- Alumno 2: Refutará a favor. Defenderá el tema y expondrá sus argumentaciones y motivaciones a favor. Aportará toda la documentación que han recopilado mediante la investigación previa de la pregunta planteada a favor. (2 min.).

- Alumno 3: Refutará en contra. Expondrá las argumentaciones y razones en contra de la pregunta a debatir recopilado mediante la investigación realizada (2 min.).

- Alumno 4: Tras las argumentaciones a favor y en contra se abre el tiempo de debate a toda la audiencia, quienes aportarán también sus argumentaciones. Durante el mismo, el cuarto alumno tendrá dos funciones: 1) Dinamizará el debate con los asistentes a clase (grupo a favor y grupo en contra) y anotará en la pizarra las nuevas aportaciones (12 min.). Es importante que los asistentes a clase puedan venir documentados y/o informados sobre el tema a debatir. Para ello podrán consultar la relación de temas y fechas de exposición en el campus virtual. 2) Expondrá las reflexiones y principales conclusiones recogidas de la participación del alumnado asistente a clase y recogido en la pizarra, enfatizando los momentos más relevantes del debate. Se formulará de nuevo la pregunta a la clase por si alguien ha cambiado de opinión una vez ampliado los conocimientos y opiniones sobre el tema con más información y argumentaciones. Se realizará de nuevo el recuento a favor y en contra, la nueva situación de la clase (2 min.).

Para la evaluación de la actividad, el profesorado participante en los distintos grupos valorará la presentación realizada a nivel individual por cada uno de los miembros del equipo y grupal en la sesión de debate, valorando: la calidad argumental y de las evidencias aportadas, la capacidad de razonamiento y respuestas, el trabajo en equipo, las habilidades de comunicación verbal y no verbal y la impresión general, además de la dinamización del debate generado en la clase (audiencia). En cuanto a las competencias y habilidades de comunicación escrita y de síntesis aplicando los conocimientos teóricos propios de la asignatura de organización y gestión de empresas se evalúa en un informe final de conclusiones que debe ser presentado a través del campus virtual. 


\section{Una experiencia de innovación docente: debates en el aula}

La experiencia que se describe en este trabajo se enmarca dentro de un proyecto de innovación docente avalado por la Universidad de Cádiz y se ha desarrollado en la asignatura “Administración de empresas” de primer curso del GRRLLy RRHH durante el curso 201718. Es una materia básica del título con 6 créditos ECTs, 4 teóricos y 2 prácticos, que se imparte en dos campus y en grupo de mañana y tarde, y con 124 alumnos matriculados, 94 de primera matrícula y 30 repetidores. Las sesiones de debate se programaron en las clases teóricas, ya que son las que venían sufriendo una mayor falta de asistencia del alumnado.

Si bien el elemento central de esta experiencia ha sido el debate académico como herramienta eficaz para el desarrollo y la mejora de las competencias analizadas, la metodología utilizada ha sido más amplia a fin de conseguir todos los objetivos propuestos. Se exponen a continuación las distintas etapas desarrolladas a lo largo del proyecto:

a. Exposición de la metodología planteada a todo el alumnado el primer día de clase, explicando detalladamente la actividad de debate. El objetivo principal en esta primera jornada es despertar el interés de los estudiantes, así como darles a conocer las competencias que se trabajarán a lo largo del curso. Según Rianudo et al (2006), el alumnado debe conocer el objetivo de todas y cada una de las actividades que se les plantea y los de la asignatura en su conjunto, es decir, cada una de las competencias asociadas a la misma.

b. Formación de equipos de trabajo con cuatro integrantes y propuesta inicial de temas por parte del alumnado. De acuerdo con las conclusiones de Pociask et al (2017), que señalan que el método para la formación de equipos parece no ser una variable relevante en su desempeño, entendemos que es interesante que el alumnado asuma la responsabilidad de elección tanto de los integrantes del equipo como el tema sobre el que quieren trabajar.

c. Como se ha comentado, uno de los objetivos planteados es la toma de conciencia por parte del alumnado de las competencias que se trabajarán en la asignatura. Para ello, se elaboró un cuestionario para la autoevaluación de las competencias específicas, generales y transversales que los estudiantes cumplimentaron durante la segunda sesión de clase. El cuestionario previo está dividido en dos bloques: el primero nos permite identifica al alumnado por nombre, sexo y grupo de clase al que pertenecen; en el segundo bloque el alumnado realiza una autoevaluación de sus competencias : 1) soy capaz de comunicarme de forma oral usando la terminología y técnica adecuada; 2) soy capaz de comunicarme de forma escrita usando la terminología y técnica adecuada; 3) soy capaz de gestionar la información correctamente; 4) soy capaz de tomar decisiones en el momento y tiempo adecuados; 5) tengo destreza a la hora de trabajar en equipo; 6) esta metodología me ayudará a ser capaz de conocer e identificar los conceptos teóricos propios de la organización y la dirección de empresa. Estos seis ítems han sido medidos con una escala Likert de cuatro puntos, a fin de evitar los sesgos de tendencia central (Hernández et al, 2001), donde 1 corresponde a nada y 4 a mucho. Este primer cuestionario lo respondieron 102 alumnos, aquellos que al principio de curso manifestaron su interés de participar en el proyecto. No obstante, el número final de participantes en la actividad de debate es de 93 estudiantes. 
d. En la segunda semana del curso se celebró una jornada cocreativa de formación impartida por un experto campeón del mundo de debate universitario. Tras ella se abre un breve plazo para la reformulación de las preguntas a debatir inicialmente propuestas, para perfilarlas una vez participado en la jornada.

e. Todos los temas y fechas para la celebración de los debates se publicaron en el campus virtual a fin de que el alumnado oyente pudiera buscar información previa y formar su propia opinión sobre el tema a debatir en cada sesión en clase.

f. Desarrollo de los debates en el aula según la organización que se ha expuesto en el epígrafe anterior.

g. Elaboración y entrega de un informe final, a través del campus virtual, con las reflexiones y conclusiones obtenidas por parte de cada equipo.

h. Al finalizar el curso, el alumnado cumplimenta un segundo cuestionario, más amplio que el anterior, en el que vuelven a realizar una autoevaluación de competencias, así como una valoración de las distintas actividades realizadas durante el curso y su grado de satisfacción con la metodología desarrollada. En este cuestionario, además de los ítems incluidos en los dos primeros bloques del primer cuestionario, valorados con la misma escala, se incluyó un tercer bloque con dos ítems sobre la valoración de los debates y sobre si recomendarían o no esta metodología para el curso siguiente, y un cuarto bloque con tres ítems sobre su satisfacción con la metodología, con su equipo de trabajo y con el trabajo realizado. En todos estos ítems, excepto el de recomendación para el curso siguiente que es una pregunta dicotómica (si/no), se ha utilizado una escala Likert de cuatro puntos (1: nada satisfecho, 4: muy satisfecho). Al final se incluyen una pregunta abierta para que indiquen qué otras cosas creen han aprendido. Este segundo cuestionario fue cumplimentado por 87 alumnos, número inferior al primero. Por tanto, los datos del alumnado que no contestaron al segundo cuestionario fueron eliminados del primero.

i. A lo largo del curso se pasó lista para constatar la asistencia a clase en las sesiones de debate.

\section{Resultados}

En este epígrafe se exponen los resultados derivados del análisis de los datos que se han obtenido a partir de los dos cuestionarios realizados, de los registros de asistencia a clase y de las calificaciones finales obtenidas por los estudiantes y recogidas en las actas definitivas. Para el tratamiento estadístico de esta información se ha utilizado la hoja de cálculo Excel y los paquetes estadísticos SPSS (versión 25) y R (paquete ggplot2).

\section{Autoevaluación de competencias}

En primer lugar, se exponen los resultados relacionados con el primer objetivo planteado relacionado con el desarrollo y mejora de las competencias específicas, generales y transversales de los estudiantes. Para el análisis de la información se han comparado las autoevaluaciones de los estudiantes en las seis competencias analizadas antes y después del desarrollo de la actividad de debate propuesta. Los resultados reflejan un aumento considerable en el porcentaje de alumnos que consideran que poseen "bastante" o "mucho" de cada una de las competencias generales y transversales una vez terminado el curso, siendo dicho incremento del 29,5\% para la competencia C1, del 14,3\% para C2, 10,3\% para C3 y

No 8, 2020. Página $\mid 70$ 
del 15,2\% para C4. En el caso de la competencia C5, si bien este incremento es menos significativo (1,6\%), sí que hay que destacar el aumento del número de estudiantes que consideran poseer "mucho" de esta competencia, pasando de representar el 22,5\% al 41,8\%. En la competencia específica C6 se ha producido una reducción de dicho porcentaje en un $3,7 \%$. No obstante, es la competencia en la que los estudiantes muestran unos valores más altos en la autoevaluación. Los datos se muestran en el gráfico 1.

Gráfico 1: Resultados autoevaluación de competencias en porcentajes

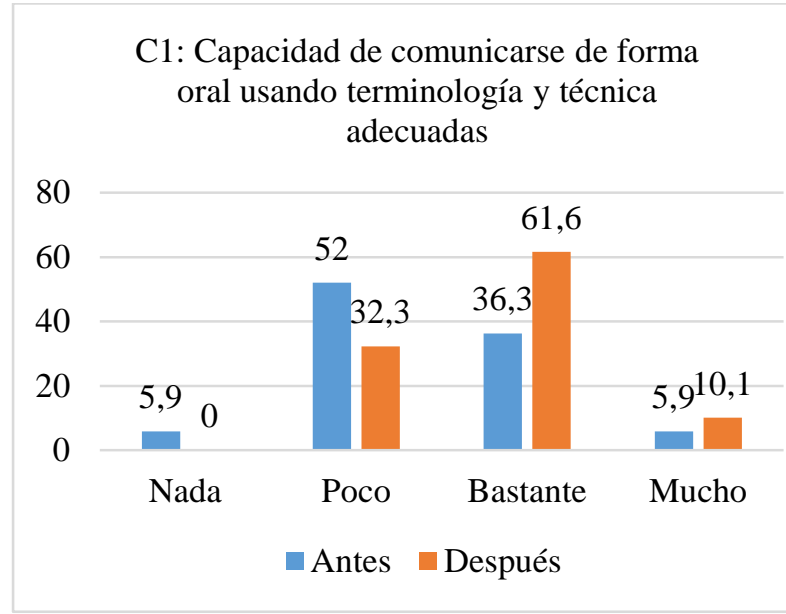

C2:Capacidad para comunicarse de forma escrita usando terminología y técnica adecuadas
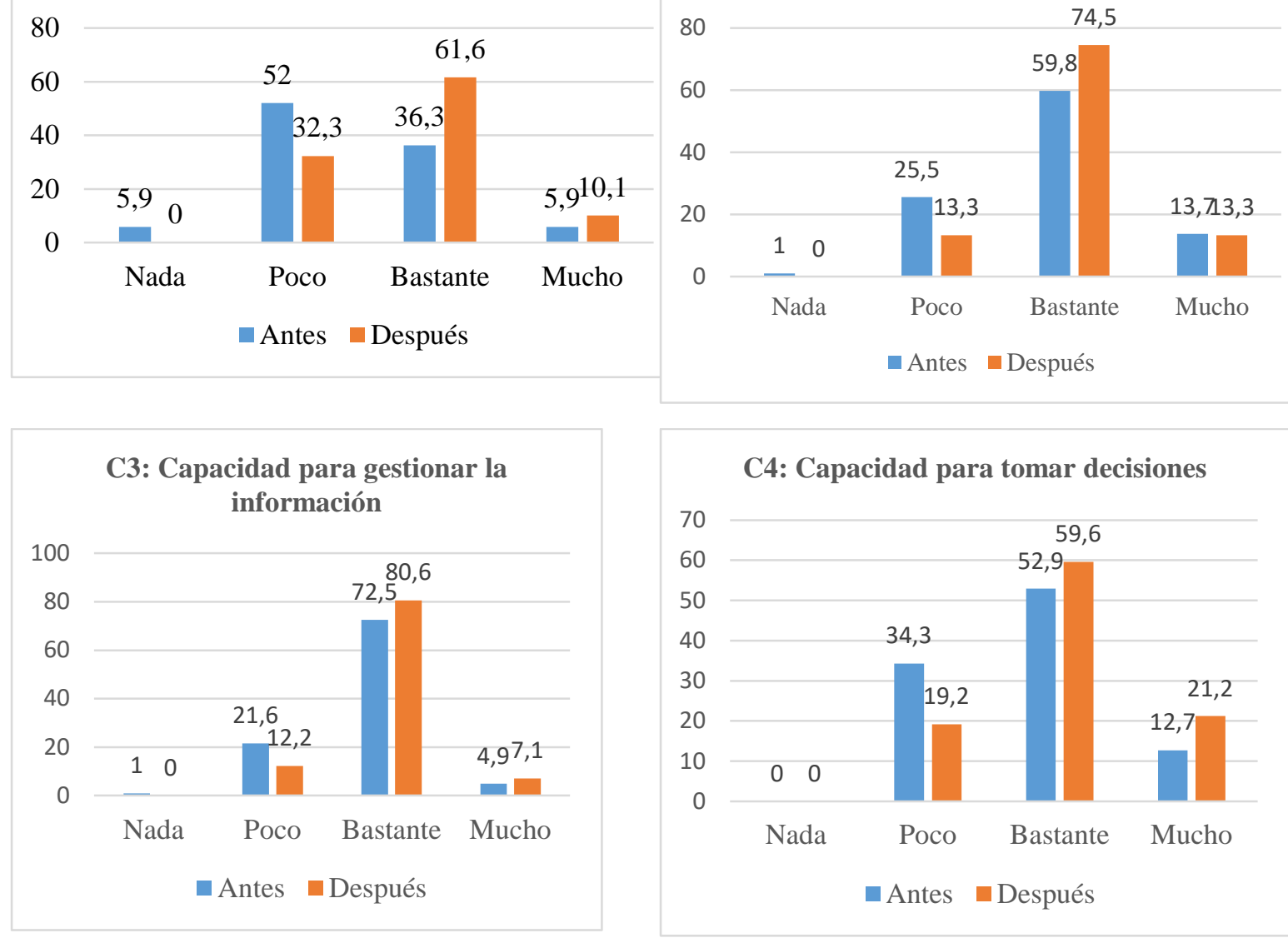

C4: Capacidad para tomar decisiones

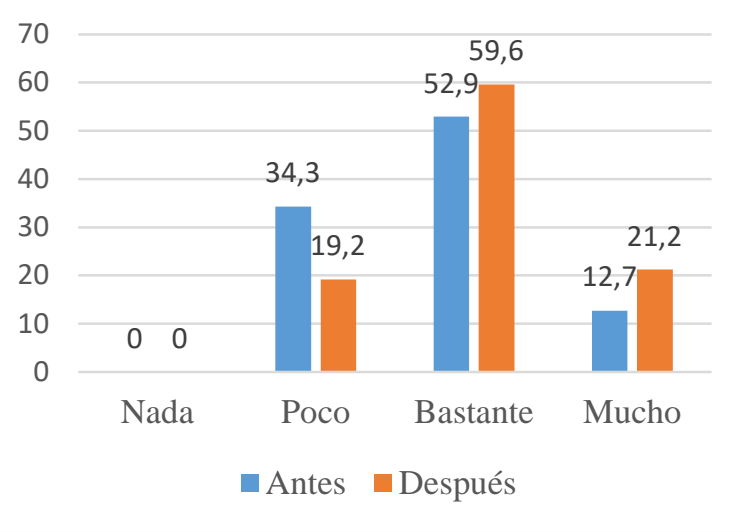


C5: Destreza para trabajar en equipo

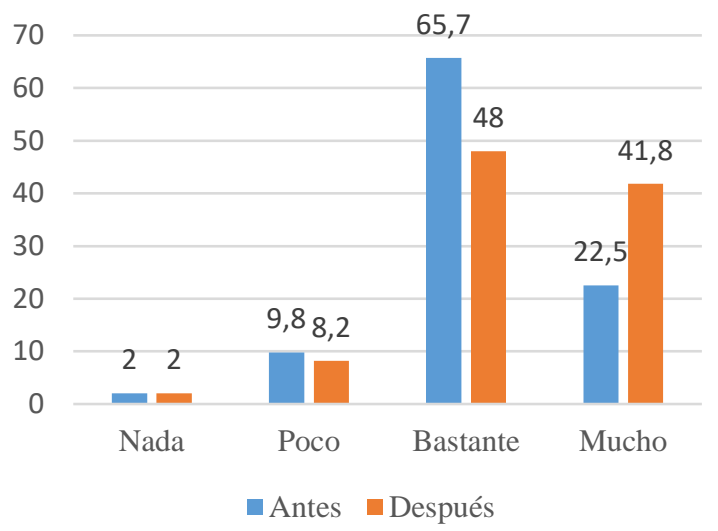

C6: Conocer e identificar los conceptos teóricos propios de la organización y dirección de empresas

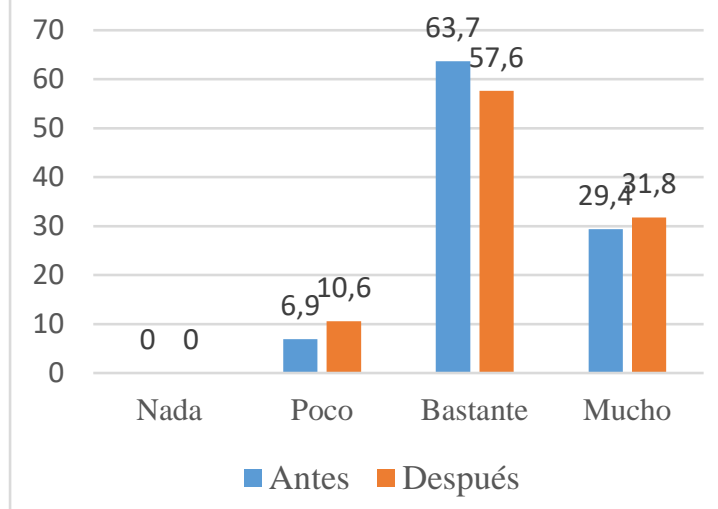

Fuente: Elaboración propia

A fin de confirmar estos resultados, utilizando el test t-Student se ha contrastado la hipótesis de igualdad de medias para datos pareados comparando la percepción inicial con la final. Dicho análisis da como resultados incrementos positivos en todas las competencias, salvo en la C6 donde los cambios son mínimos, siendo significativamente importante solo en las competencias C1 (p-valor $=0.00005)$ y en la C4 (p-valor=0.007).

\section{Tasas de éxito y rendimiento}

Se ha realizado en primer lugar un análisis de las tasas de éxito y rendimiento globales de la asignatura, para lo que se han calculado dichas tasas considerando, por un lado, a todo el alumnado matriculado y, por otro lado, solo el grupo de estudiantes participantes en la actividad de debate. Según se observa en la tabla 2, las tasas que presentan el grupo de participantes en el proyecto son superiores que las obtenidas por el total de alumnos matriculados, siendo los porcentajes de incremento en la tasa de éxito de un 2,55\% y de un $10,26 \%$ en la de rendimiento.

Tabla 2: $\mathrm{N}^{\mathrm{o}}$ de alumnos y tasas de éxito y rendimiento

\begin{tabular}{|l|c|c|}
\cline { 2 - 3 } \multicolumn{1}{c|}{} & Matriculados & Participantes en debates \\
\hline Total & 124 & 93 \\
\hline Aprobados & 52 & 50 \\
\hline Presentados & 98 & 87 \\
\hline Tasa éxito & $53,06 \%$ & $57,47 \%$ \\
\hline Tasa rendimiento & $41,94 \%$ & $53,76 \%$ \\
\hline
\end{tabular}

Fuente: Elaboración propia 
Leyenda: La tasa de éxito es la relación entre el total de aprobados respecto a los presentados y la tasa de rendimiento la relación entre el número de aprobados y los matriculados.

Si bien los datos anteriores reflejan una mejora en las tasas de rendimiento y éxito globales de la asignatura, nos interesa conocer si aquellos estudiantes que han participado en la actividad de debate han obtenido mejores calificaciones finales en la misma. Los resultados del test Chi-cuadrado muestran una dependencia significativa entre la participación en la actividad y la calificación final obtenida en la asignatura. Los datos obtenidos del estadístico están recogidos en la tabla 3.

Tabla 3. Pruebas Chi-cuadrado

\begin{tabular}{lr|r|r} 
& Valor & Df & $\begin{array}{c}\text { Significación } \\
\text { asintótica } \\
\text { (bilateral) }\end{array}$ \\
\hline $\begin{array}{l}\text { Chi-cuadrado de } \\
\text { Pearson }\end{array}$ & $41,818^{\mathrm{a}}$ & 3 &, 000 \\
\hline Razón de verosimilitud & 42,050 & 3 &, 000 \\
\hline N de casos válidos & 124 & & \\
\hline
\end{tabular}

Fuente: Elaboración propia

Leyenda a. 2 casillas (25,0\%) han esperado un recuento menor que 5. El recuento mínimo esperado es 1,50 .

En el gráfico 2 queda reflejada la distribución de notas entre los estudiantes que participaron en la actividad de los debates y los que no lo realizaron, apreciándose calificaciones más altas entre aquellos que sí decidieron realizarlo. 
Gráfico 2. Diagrama de densidad de notas finales según la participación en debates

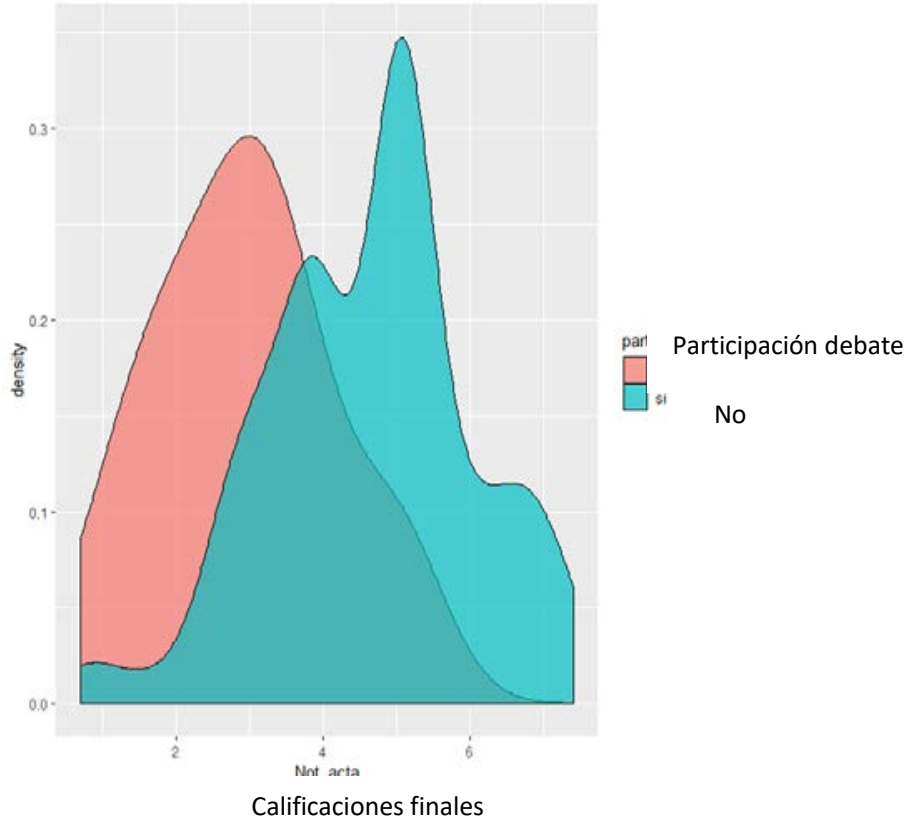

Fuente: Elaboración propia

En el diagrama de caja y bigotes con nubes de puntos modificada (gráfico 3), se muestra tanto la distribución de los valores como las medias de las notas obtenidas (punto rojo) por el alumnado según participara o no en los debates, dónde se aprecia cómo la nota media es superior en el alumnado que realizó la actividad de debate.

Gráfico 3: Diagrama de caja y bigote con nubes de puntos con media

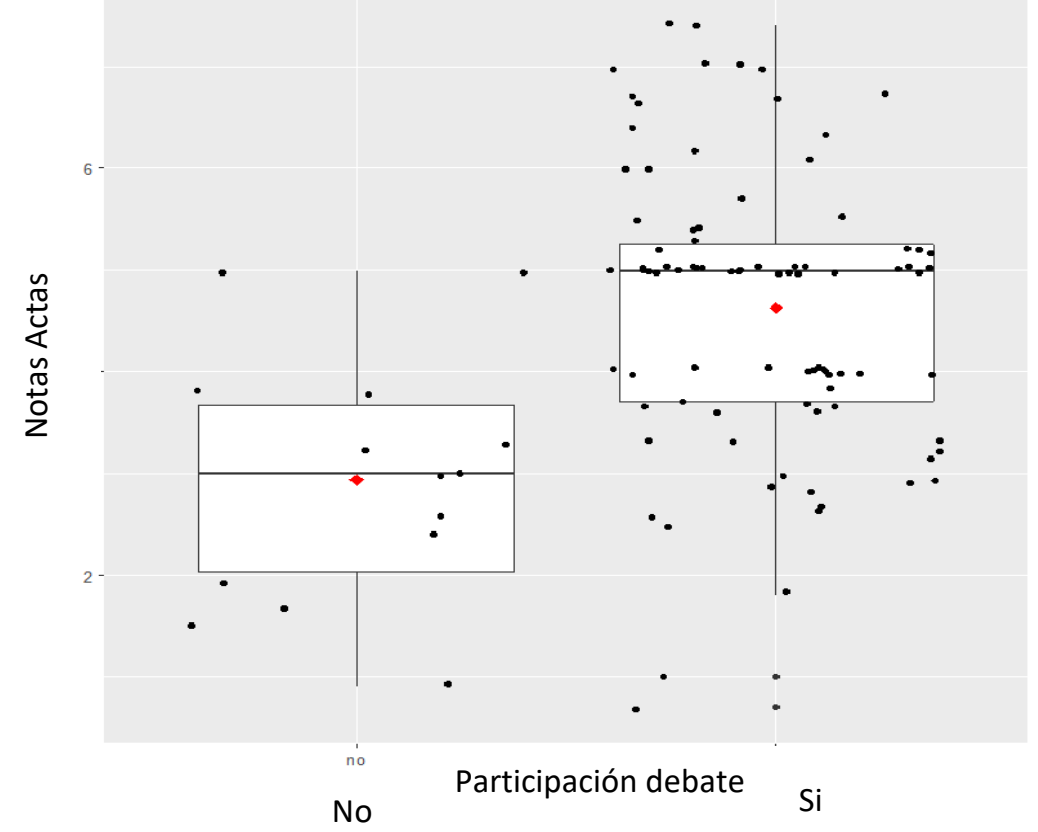

Fuente: Elaboración propia 
Desde un punto de vista descriptivo hay una apreciable diferencia entre la nota media obtenida por los estudiantes que han participado en los debates $(4,626)$ con la que obtuvieron los que no lo realizaron (2,936). Incidiendo en esta dirección, si se comparan mediante el contraste de la t-Student para muestras independientes las notas medias obtenidas por el grupo de estudiantes participantes con los no participantes se obtiene como resultado que la calificación media entre los que han participado es significativamente mayor que la de los que no lo han realizado (p-valor=0), ver tabla 4.

Tabla 4. Estadísticas de grupo

\begin{tabular}{|c|c|c|c|c|c|}
\hline & $\begin{array}{l}\text { Participación en los } \\
\text { Debates }\end{array}$ & $\mathrm{N}$ & Media & $\begin{array}{c}\text { Desv. } \\
\text { Desviación }\end{array}$ & $\begin{array}{c}\text { Desv. Error } \\
\text { promedio }\end{array}$ \\
\hline \multirow{2}{*}{$\begin{array}{l}\text { NOTA_AC } \\
\text { TA }\end{array}$} & No participó & 14 & 2,936 & 1,2226 & ,3268 \\
\hline & Participó & 87 & 4,626 & 1,3934 & 1494 \\
\hline
\end{tabular}

Prueba de muestras independientes

Prueba t para la igualdad de medias

\begin{tabular}{|c|c|c|c|c|c|c|c|}
\hline & \multirow[b]{2}{*}{$\mathrm{t}$} & \multirow[b]{2}{*}{ gl } & \multirow{2}{*}{$\begin{array}{c}\text { Sig. } \\
\text { (bilateral } \\
\text { ) }\end{array}$} & \multirow[b]{2}{*}{$\begin{array}{l}\text { Diferencia } \\
\text { de medias }\end{array}$} & \multirow{2}{*}{$\begin{array}{c}\text { Diferenci } \\
\text { a de error } \\
\text { estándar }\end{array}$} & \multicolumn{2}{|c|}{$\begin{array}{l}95 \% \text { de intervalo de } \\
\text { confianza de la } \\
\text { diferencia }\end{array}$} \\
\hline & & & & & & $\begin{array}{l}\text { Inferi } \\
\text { or }\end{array}$ & Superior \\
\hline $\begin{array}{l}\text { NOTA_ACT } \\
\text { A }\end{array}$ & $-4,279$ & 99 & 0,000 & $-1,6907$ & 0,3951 & $\begin{array}{r}- \\
2,474 \\
8\end{array}$ & $-0,9067$ \\
\hline
\end{tabular}

Fuente: Elaboración propia

\section{Asistencia a clase}

En cuanto a la asistencia a clase, se ha ido registrando la asistencia de forma nominativa en todas las clases impartidas a lo largo del curso. Los datos muestran unas tasas de asistencia razonablemente buenas, alrededor del 60\% del alumnado asiste regularmente a clase, tanto a las clases prácticas como a las teóricas, manteniéndose esos niveles a lo largo de todo el curso.

\section{Valoración de la experiencia por parte del alumnado}

Por último, se exponen los datos obtenidos de la valoración y evaluación que realizan el alumnado de la metodología, el equipo y el trabajo realizado a partir de la información obtenida de los cuestionarios. El análisis descriptivo de los datos indica una alta satisfacción 
del alumnado. El 95\% señala estar satisfecho o muy satisfecho con la metodología empleada, y el 91,9\% con su equipo y con el trabajo realizado. Además, el 95,9\% dice recomendar esta actividad para el curso siguiente. La tabla 5 muestra los resultados obtenidos.

Tabla 5: Grado de satisfacción con la metodología, el equipo y el trabajo realizado

\begin{tabular}{|l|c|c|c|c|}
\cline { 2 - 5 } & $\begin{array}{c}\text { Nada } \\
\text { Satisfecho }\end{array}$ & $\begin{array}{c}\text { Poco } \\
\text { Satisfecho }\end{array}$ & Satisfecho & $\begin{array}{c}\text { Muy } \\
\text { Satisfecho }\end{array}$ \\
\hline $\begin{array}{l}\text { Grado de satisfacción con } \\
\text { la metodología utilizada }\end{array}$ & $1 \%$ & $4 \%$ & $68,7 \%$ & $26,3 \%$ \\
\hline $\begin{array}{l}\text { Grado de satisfacción con } \\
\text { el equipo }\end{array}$ & $0 \%$ & $8,1 \%$ & $42,9 \%$ & $49 \%$ \\
\hline $\begin{array}{l}\text { Grado de satisfacción con } \\
\text { el trabajo realizado }\end{array}$ & $0 \%$ & $8,1 \%$ & $56,6 \%$ & $35,3 \%$ \\
\hline
\end{tabular}

Fuente: Elaboración propia

\section{Conclusiones}

Se presentan a continuación las principales conclusiones que se desprenden del análisis realizado. En primer lugar, la utilización del Debate Académico ha demostrado ser una práctica facilitadora de determinadas competencias transversales, genéricas, y especificas enmarcadas en la asignatura de Administración de Empresas, del Grado de RR.LL. y RR.HH., entre ellas: expresión oral y escrita usando técnicas y terminologías adecuadas, búsqueda y selección de información, trabajo en equipo, síntesis y capacidad de respuesta. Se podría considerar adecuada, por tanto, su implementación como herramienta docente, así como una vía alternativa en la adquisición de conocimientos teóricos de la asignatura.

El alumnado participante en la experiencia, no solo ha conocido y reconocido las competencias específicas, transversales y generales analizadas, sino que manifiestan haber mejorado el grado en que poseen dichas competencias, especialmente la comunicación oral y la capacidad de tomar decisiones. Sin embargo, esto no ha ocurrido con la competencia específica relacionada con los conceptos propios de la organización y administración de empresas. Probablemente esto se deba a que, si bien todos los temas propuestos tienen su vertiente empresarial, no en todos los casos los participantes la han identificado de una forma clara. Al alumnado le cuesta normalmente ir más allá del contenido propuesto en la programación de la asignatura, con un enfoque de "lo que entra en examen” y la actividad del debate les sirve para enriquecer su enfoque hacia un cambio conceptual que permita conectar la experiencia personal, los conocimientos y las contingencias del entorno.

En segundo lugar, se puede señalar que la metodología basada en el debate académico ha despertado gran interés entre el alumnado, no solo por el elevado número de estudiantes participantes sino por la calidad de los temas propuestos y debatidos y por los argumentos 
esgrimidos en el mismo. De hecho, la nota media que han obtenido los equipos en esta actividad es de 7,3 puntos sobre 10.

En tercer lugar, el alto interés mostrado por los estudiantes también se ha visto reflejado en las tasas de asistencia y participación en clase, que se han mantenido a lo largo del curso. Esto es un aspecto diferenciador respecto a años anteriores en los que al final del curso se venía produciendo un importante descenso. Esta mayor asistencia a clase, unido al buen desempeño mostrado por los estudiantes en la actividad de debate, ha influido positivamente en las calificaciones finales obtenidas por los estudiantes en la asignatura Administración de Empresas, siendo mayores en aquellos que han participado en la actividad de debate. Esto ha supuesto a su vez, un incremento en las tasas de éxito y rendimiento globales de la misma, en consonancia con lo expuesto por Rodríguez y Herrera (2009) y Bernardo et al (2015).

En cuarto lugar, hay que resaltar la gran satisfacción del alumnado participante tanto con la metodología utilizada, como con su equipo de trabajo y con el desempeño del mismo, siendo altamente recomendada por ellos como una actividad para el curso siguiente. Los resultados obtenidos confirman la necesidad de cambiar nuestras metodologías de clase en orden a una mayor intervención del alumnado en la construcción de su propio aprendizaje y en la toma de conciencia por parte de este de la relación positiva existente entre la asistencia a clase y el rendimiento.

Por último, destacar que el alto grado de satisfacción mostrado por el alumnado con la experiencia, se traduce también en una alta satisfacción de las docentes implicadas en ella, a lo que añadimos la motivación e inquietud por mejorar nuestra labor docente y apostar por una enseñanza universitaria abierta, adaptada a los tiempos y conectada con la actualidad de su entorno específico y genérico, lo que nos lleva a seguir trabajando en esta misma línea en próximos cursos. No obstante, el proceso de aplicación y puesta en marcha de la metodología, así como las opiniones de los alumnos recogidas en los cuestionarios y las conclusiones extraídas del análisis de los resultados obtenidos, nos permiten identificar y plantear una serie de mejoras para el futuro y que se exponen a continuación.

\section{Propuestas de mejora}

La primera de las propuestas de mejora proviene de la pregunta abierta que se había incluido al final del segundo cuestionario sobre qué otras cosas entienden los alumnos que han aprendido y que ha tenido respuestas muy interesantes. En concreto, el alumnado ha manifestado que la experiencia de los debates les ha ayudado a perder el miedo a hablar en público, a mejorar su expresión, y a empatizar más con las ideas de otros. Por ello, entendemos que en una próxima edición se debe profundizar en el desarrollo de esas otras competencias transversales, especialmente en la argumentación y en el fomento del espíritu crítico, elementos fundamentales para una formación global de los universitarios, así como una mejor conexión entre los temas tratados y la vertiente empresarial de los mismos.

Por otro lado, los temas tratados y debatidos muestran una gran sensibilidad del alumnado hacia temas sociales y medioambientales, en línea con las nuevas demandas y sensibilidades de la sociedad en su conjunto. Por ello, como segunda propuesta de mejora creemos que es importante incidir en la toma de conciencia, por parte del alumnado, de su papel fundamental 
como agente de cambio social. El debatir temas actuales controvertidos de carácter social, medioambiental y económico de repercusión mundial, puede ayudar al alumnado a entender mejor los conocimientos de la asignatura y a movilizar su motivación hacia el aprendizaje y conocimientos fuera de las situaciones de examen, y así reconozcan que lo que aprenden con esta metodología es útil para ellos fuera de clase. Por ello, entendemos que las preguntas o temas a debatir en el futuro podrían enmarcarse en la Agenda 2030 (ONU, 2015), en este plan de acción mundial a favor de las personas, el planeta y la prosperidad, basado en 17 Objetivos de Desarrollo Sostenible (ODS), que tiene por objeto asegurar el progreso social y económico sostenible en todo el mundo y fortalecer la paz universal dentro de un concepto más amplio de la libertad, así el alumnado podría implicarse y ser protagonista de los cambios sociales, económicos y medioambientales que propone, y relacionarlos con el contenido de la asignatura y la repercusión en su entorno.

\section{Referencias}

Ahumada, R.M., Baratas, I., Merida, D. (2015). “El “Debate Universitario” en la Práctica de la Asignatura Gestión de Empresas II del Grado de Relaciones Laborales y Recursos Humanos de la Universidad del País Vasco”. Trabajo (33), 45-71.

Bernardo, A., Cerezo, R., Rodríguez-Muñiz, L.J., Núñez, J.C., Tuero, E. y Esteban, M. (2015). "Predicción del abandono universitario: variables explicativas y medidas de prevención”. Revista Fuentes, (16), 63-84.

Facultad de Ciencias del Trabajo. (2019). Memoria Acreditada del Título de Grado en Relaciones Laborales y Recursos Humanos. Recuperado en: https://cutt.ly/BypsxyR [04 ene 2020]

Facultad de Ciencias del Trabajo. (2019). Autoinforme de Seguimiento del Título de Grado en Relaciones Laborales y Recursos Humanos, Curso 17-18. Recuperado en: https://cutt.ly/1ypsbuF [04 ene 2020]

Hernández, A., Espejo, B., González, V. y Gómez, J. (2001). “Escalas de respuesta tipo Likert ¿es relevante la alternativa “indiferente”? Metodología de Encuestas, 3 (2), 135-150.

Leitáo, S., de Chiaro, S., Cano, M. (2016). “El debate crítico. Un recurso de construcción del conocimiento en el aula”. Textos, Didáctica de la Lengua y de la Literatura (73), 2633.

Marqués, P. (2001). “Algunas notas sobre el impacto de las TIC en la universidad”. Educar, (28), 83-98.

ONU (Organización de Naciones Unidas). (2015). Objetivos de Desarrollo Sostenible, Agenda 2030. Recuperado en: https://cutt.ly/2ypsQng [04 ene 2020] 
Pociask, S., Gross, D. \& Shih, M. Y. (2017). Does Team Formation Impact Student Performance, Effort and Attitudes in a College Course Employing Collaborative Learning? Journal of the Scholarship of Teaching and Learning, 17(3), 19-33.

Rinaudo, M. C., de la Barrera, M. L., Donolo, D. (2006). Motivación para el aprendizaje en alumnos universitarios. Revista electrónica de motivación y emoción, 9(22), 1-19.

Rodríguez, C., Herrera, L. (2009). “Análisis correlacional-predictivo de la influencia de la asistencia a clase en el rendimiento académico universitario. Estudio de caso en una asignatura”. Profesorado. Revista de Currículum y Formación de Profesorado, 13(2), 1-13.

Sánchez, G. (2007). "El debate académico en el aula como herramienta didáctica y evaluativa” IV Jornadas Internacionales de Innovación Universitaria. Métodos docentes afines al EEES. 12 y 13 de julio, Universidad Europea de Madrid. 